\title{
Opportunities for innovation in neutron activation analysis
}

\author{
Peter Bode
}

Received: 16 May 2011/Published online: 3 June 2011

(c) The Author(s) 2011. This article is published with open access at Springerlink.com

\begin{abstract}
Neutron activation laboratories worldwide are at a turning point at which new staff has to be found for the retiring pioneers from the 1960s-1970s. A scientific career in a well-understood technique, often characterized as 'mature' may only be attractive to young scientists if still challenges for further improvement and inspiring new applications can be offered. The strengths and weaknesses of neutron activation analysis (NAA) are revisited to identify opportunities for innovation. Position-sensitive detection of elements in large samples, Monte Carlo calculations replacing the use of standards, use of scintillator detectors and new deconvolution techniques for increasing the sensitivity are examples of challenging new roads in NAA. Material science provides challenges for the application of NAA in both bulk samples, ultrathin layers and ultrapure materials.
\end{abstract}

Keywords Neutron activation analysis - Innovation . Large samples · Position-sensitive $\cdot$ Scintillator .

Material science $\cdot$ Metrology

\section{Introduction}

Neutron activation analysis (NAA) is often characterized as a "mature" technique. Probably the first such denotation came from the 1970 bi-annual review "Nucleonics" in the Analytical Chemistry Journal [1]:

\section{P. Bode $(\square)$}

Department of Radiation, Radionuclides and Reactors, Faculty of Applied Sciences, Reactor Institute Delft, Delft University of Technology, Mekelweg 15, 2629 JB Delft, The Netherlands e-mail: p.bode@tudelft.nl
...In writing our first review four years ago, we started our intention to restrict ourselves to "items that appear original, novel or potentially useful". We continue to use this guideline, but in a period marked by considerable disenchantment with hard science, particularly the physical and chemical, and most particularly those aspects of it which seem to bear little relation to current pressing social problems, it is not surprising that we find a smaller number of papers that meet our criteria. It appears that nucleonics has reached maturity and will probably yield fewer startling innovations than in the past. Certainly many applications and routine methods continue to be published and it is, we think, an indication of the vitality of the field that these occur. But the innovative progress of most tracer applications, measurement techniques, and radioanalytical methods has been slower and less spectacular than one might wish. This is true for neutron activation also; both generator and reactor applications have increased, but that which is original and of non-routine interest has appeared primarily in the fields of charged particle and nuclear reaction analysis...

"Mature" can be interpreted as "completed in development", a stage in which the initial problems have been overcome. The trueness of Lyon et al.'s statement is underpinned by the classical book of De Soete et al. [2] which was published in 1972 - and hence may have already been compiled at least 1-2 years earlier. The developments in the years after can be categorized as refinements of the knowledge already existing in 1970, most of it already laid down in this book. The single comparator method, forming the basis of the $k_{0}$ method of standardization, dates back to 1965 [3]; almost all current gamma-spectrum analysis 
methods are similar in nature as SAMPO, published in 1969 [4]. The same applies to Compton suppression counting [5], and epithermal NAA [6]. Real innovations building on NAAs methodological strengths and leading to breakthroughs in its weaknesses have not been ample since 1970. An exception could be made for the prompt-gamma method [7], high-count rate processing systems [8], neutron depth profiling [9] and the expansion of the capabilities towards the analysis of large, irregular shaped objects [10]. Seventy-five years after Hevesy and Levi did the experiments that are marked as the birth of NAA it can be concluded that the principles of the technique are well understood, forming the basis for being designated as a primary method of measurement [11].

Indeed, as foreseen by Lyon et al. in 1970, the vitality of NAA has been demonstrated by the large number of applications. One could criticize if all applications were build on the strengths of NAA but a similar comment can also be addressed to the applications of other methods for element determination. Worldwide, a dozen of NAA laboratories have been successful in attaining ISO/IEC17025 accreditation which is an indication that analyses can be performed with adequate technical competence and of direct economical and (inter)national relevance. However, this is a small fraction (estimated about 10\%) of all NAA laboratories in the world.

Now, 40 years later after Lyon's comments, NAA laboratories worldwide are facing viability challenges, especially with respect to staffing as many that pioneered the technique in the $1960 \mathrm{~s}$ and $1970 \mathrm{~s}$ are retiring. Many organizations are missing strategies for timely replacement of retiring staff by appointing successors well before the retirement takes place. The absence of such a strategyand thus of potential vacant positions-has a negative effect on the attractiveness to young academics of career making in NAA and its applications. It eventually hampers the expansion of the utilization due to the lack of experienced and creative manpower.

An outlook for innovative research opportunities is often the major driving force for young academics to select a field of science for career making, as they may wish to exploit their creativity under optimal conditions. NAA laboratories need creative staff with fundamental radiochemical/analytical/nuclear physics background to anticipate on new fields of application; preferably those that may generate additional funding. The question thus arises: does NAA can still advertise itself being a science with challenging innovative research on the principles and applications of the method, in such a way that will invite new people to step into it? To this end, the strengths and weaknesses of NAA should be revisited to identify the areas to focus on further expanding on the strengths and innovations to compensate for the weaknesses.
Strengths and weaknesses of NAA

Methodological strengths and weaknesses should in principle be separated from the intended application of a technique. Material to be analyzed, element(s) of interest and element(s) yielding interfering nuclear reaction product(s), and their amounts; the desired degree of trueness, precision and turnaround time-and some times also the analysis' costs - all are decisive factors contributing to the final conclusion. However, the methodological strengths are essential in identifying potential fields of application and prioritizing this on basis of their social/economical/ scientific relevance. Research, focused on innovations specific for further powering and expanding these strengths will form the challenge for many NAA laboratories.

The main strength of NAA is that it is physically fully described and understood. All potential interferences, sources of error and contributions to uncertainty of measurement are known, and can be quantified [11]. (N)AA is the only technique for qualitative and quantitative element determination based on phenomena occurring in the atomic nucleus. As such, the technique distinguishes to other techniques by the absence of effects of chemical binding to the trueness of results. ${ }^{1}$ Various other characteristics such as high element specificity, multi-element determination ability, and predictable sensitivity are all consequential from the metrological completeness of the technique.

The second strength of NAA is that there is no fundamental requirement on the size, shape and chemical state (solid or liquid) of the test portion. Moreover, the test portion may be even kept integral as received. This is often translated into the non-destructive analytical characteristic of NAA. The high penetrating depths of neutrons and gamma-rays allow for analysis of bulk quantities by which it differs from XRF which is a surface analysis technique.

Other techniques can handle solids directly too, such as PIXE, laser ablation methods (LA-ICP, LIBS) and solid state AAS though in all cases only very small portions are characterized.

The third strength of NAA is that the neutron irradiation of the main constituents of many materials such as $\mathrm{H}, \mathrm{C}, \mathrm{O}$, $\mathrm{N}, \mathrm{Si}, \mathrm{P}, \mathrm{Ca}$, do not result in significant induced radioactivity, making the test portion transparent for the signals from the activation products of the other (trace) elements.

The physical nature of NAA also defines its main weakness. The sensitivity for determination of elements is

\footnotetext{
${ }^{1}$ One of the few exceptions to this statement is that for some radionuclides, the intensity of a sum-peak in a gamma-gamma cascade in the nuclear decay may be slightly altered by the perturbation of the angular correlation of these gamma-rays, caused by the electromagnetic field gradients of atoms surrounding the decaying nucleus. These field gradients may be different for different chemical compounds [12].
} 
largely set by a physical parameter, the activation cross section, and the characteristics of the decaying activation product such as half-life, type, energy and intensity of radiation emitted. Since the sensitivity is different for different elements, optimization protocols for determining an element in the presence of other elements may require the introduction of lengthy decay times after irradiation, resulting in the often quoted long turn-around times.

Similarly, the strength of being a technique based on phenomena occurring in the atomic nucleus defines its second weakness: NAA does not provide information on the chemical binding/speciation of elements. Moreover, the irradiation and subsequent recoil and decay process may cause such a bond rupture that the link between the decaying nucleus and its original speciation will disappear anyhow.

\section{Opportunities for innovations}

\section{Building on strengths}

For long, NAA has been promoted as a 'non-destructive' technique, with excellent capabilities for analysis of solid material. Typical sample sizes were for practical reasons often limited to less than $1 \mathrm{~g}$. With the development of high powered microwave digestions systems coupled to techniques like ICP and AAS, as well as by improvement of correction methods in XRF, also other techniques now can relatively easy process solid materials with quantities up to a few grams. But, being a technique based on very well known physical principles, NAA has the unique capability of analyzing much larger the amounts than applied in any other technique [10], varying from $1 \mathrm{~g}$ to the kilogram range. This puts NAA in the unique position of analyzing directly-without the risk of element losses and contamination during sample size reduction-of samples of the minimum sample mass, as prescribed by the sampling theory for rendering a pre-defined minimum variance of the property of interest. Often masses up to several tens of grams may already suffice. It provides also the opportunity for an experimental verification of the validity of such sampling theories or sampling constants, and of the validity of results obtained by micro-analytical techniques.

This large sample capability applies both the 'normal' NAA as well as to prompt-gamma NAA [13]. Moreover, in both approaches there are no fundamental limitations to the shape of a large test portion. Any arbitrary shaped sample can be irradiated with neutrons and the induced radioactivity be measured. Quantitation on basis of internal standards has already been demonstrated [14]; alternatively duplicated phantoms of known composition may equally render satisfying results [15].
Collimated scanning of the activity of large test portions, the use of focused neutrons in prompt gamma-NAA offers the opportunity of 2-dimensional (2D) bulk trace element determinations for the detection of local inhomogeneities [16]. In principle, there are no fundamental limitations for even 3-dimensional (3D) mapping of the element content of large test portions especially in a hybrid set-up with CT scanning and/or neutron tomography. There may remain limits to the spatial resolution, but any $3 \mathrm{D}$ distribution profile may be of added value above none at all and/or the alternative of analyzing subsamples.

Technically, challenges can be found in the design of irradiation facilities, correction methods for changes in the neutron energy distribution inside the large test portion once irradiations are carried our close to the reactor core; and in the $3 \mathrm{D}$ reconstruction of the quantitative element distribution. The traditional expression of element content as 'mass fraction' includes an assumption of perfect degree of homogeneity, which in principle does not apply to large samples. Hence, new metrological concepts are needed for estimating and expressing the degree of trueness for inhomogeneous materials.

Monte Carlo modeling of the neutron energy distribution in an irradiation facility, for estimation of the reaction rate as well as Monte Carlo calculation of detection efficiency offers an outlook for standardless NAA [17, 18]. Challenges may be found with the further perfectioning thereof including neutron and gamma-ray sample self-attenuation and even flux depression effects, and with accommodating changes in the neutron spectrum due to changes in the control rod position, fuel and reflector burn-up effects as well as horizontal and vertical neutron flux gradients. Eventually, this may open the door for routine application of standardless absolute NAA with an almost equivalent degree of accuracy as now can be achieved in e.g. $k_{0}$ NAA. It will introduce a significant reduction in calibration, processing time and costs, especially if combined with intelligent spectrum analysis software.

The third strength identified, viz. the limited activation of several elements, often being the major ones in many materials, has already exhaustively exploited. However, it remains a guiding factor for selecting the optimal opportunity for applying NAA.

\section{Overcoming weaknesses}

Sensitivity in NAA is, under given conditions, the ratio of measured radioactivity and mass. It is an independent physical parameter of each radionuclide in NAA, which differentiates it from the limit of detection which is often erroneously quoted as an indication of the sensitivity. The limit of detection is, since it is the signal to noise ratio, 
dependent on the sensitivity of both the measure and the noise, resulting from the sensitivities of all other activated products in the test portion.

With respect to the sensitivity, the measured radioactivity can be further differentiated towards on the interplay of neutron dose (fluence rate and irradiation duration), decay time, effective activation cross section, full energy photopeak efficiency and counting time. Assuming that an NAA laboratory with access to specific facilities has not an opportunity to significantly increase the neutron fluence rates, then, at a given induced radioactivity (based on neutron dose and effective activation cross section), the available variables to the sensitivity are decay and counting time, and full energy photopeak efficiency.

Measurement of the induced radioactivity is done very inefficiently in NAA. The absolute photopeak efficiency varies from ca. $85 \%$ at $100 \mathrm{keV}$ to ca. $10 \%$ at $1,332 \mathrm{keV}$ in the largest commercially available well-type Ge detectors (active volume ca. $250 \mathrm{~cm}^{3}$ ); but as soon as samples are counted at a few centimeters from the end cap of a Ge detector-as is needed if the sample is relatively large, see above-these values drop to the order of $1-5 \%$ at low energies and $0.1 \%$ or even lower at high photon energies. Consequently, research into further increasing the counting efficiency in NAA is worth to considering.

The new generation of high resolution scintillation detectors $\left(\mathrm{LaBr}_{3}\right.$ or $\left.\mathrm{CeBr}_{3}\right)$ offer a promising outlook because of the recent advances in spectrum deconvolution techniques for scintillation spectra $[19,20]$. The developments could make these detectors an excellent choice for applications resulting in relatively simple gamma-ray spectra. The new scintillation detectors are now available with maximum sizes up to $3^{\prime \prime} \times 3^{\prime \prime}$ which is still considerable smaller than with $\mathrm{NaI}(\mathrm{Tl})$ (e.g., like $12^{\prime \prime} \times 12^{\prime \prime}$ ). The analytical opportunities of such very large $\mathrm{NaI}(\mathrm{Tl})$ detectors, particularly in well-type configuration or as a twinned set-up (realizing almost 4 pi geometry) are also worth studying, since absolute photopeak efficiencies up to about $75 \%$ for the $1,332 \mathrm{keV}$ can be obtained [21]. Given the very high peak-to-total ratio (approaching unity for all energies), such spectra are virtually without Compton continuum. Spectrum distortion by coincidence summing effects can always be corrected for by calibration. The fast pulse rise time of the new scintillators make them suitable for high count rate processing, which makes it possible to detect very short half life radionuclides in the presence of dominating long half-life nuclides. The challenge lies in further perfectioning these new deconvolution techniques.

Whereas sensitivities may thus be increased, limits of detection may increase too albeit with approximately the square root of the improvement in sensitivity.

\section{Opportunities for applications}

NAAs position in chemical metrology has been strengthened after its designation by the CCQM in 2007 as a primary ratio method of measurement [11, 22]. More specifically, this relates to the use of NAA for the characterization of candidate certified reference materials, and to its use in CCQM key-comparisons, the results thereof to be used in the claims of calibration and measurement capabilities (CMCs) of national metrology institutes [23]. It has been recognized that NAA might be the technique of choice for studies and projects on elements at trace and ultratrace level because of its' non-destructive nature and absence of chemical matrix effects as was already recently shown [24]. Quantification of impurities in ultrapure compounds (metals, alloys, carbon) is another challenging opportunity for NAA in view of the realization of such certified reference materials.

A broad variety of the current applications of NAA in the applied fields of science will remain, often being different for each country. Success stories in specific laboratories with demonstrated sustainability have inspired others for similar application, such as in archaeology. Other applications, such as the use of NAA in epidemiology are less easy to copy because of the considerable investment in hardware automation and data processing, essential for large scale projects in which thousands of samples must be analyzed within a short timeframe. PGNAA can provide a solution for specific element determinations in bulk materials that should remain integer at minimum induced radioactivity. However, state of the art PGNAA requires cold neutron beams which is also not easy realizable in all research reactors.

At large, the capability of non-destructive determination of (trace) impurities in (ultrapure) bulk solid material can be optimally employed in the material sciences, e.g. related to development of materials for the electronic industry, solar panels, batteries and hydrogen storage systems; new catalysts, new composites, materials used in nanotechnology, carbon based materials, plastics and even large integral final end-products. Other techniques may fail since total dissolution may not be guaranteed, contamination may occur during sample processing; because not the bulk but just surfaces are scanned, or because commutable calibrators (e.g. certified reference materials) are not available. Normal NAA, PGNAA, NDP and LS-NAA have attractive characteristics for use in material science and can provide complementary information to one another. The opportunities are not limited to bulk analyses; it has been demonstrated in the past that NAA has excellent characteristics for determination of trace impurities in this ultrathin layers as used, such as silicon wafers in the microelectronic industry [25]. NDP_being a form of 
NAA - is unique for depth distribution analyses in material science. NAA has, also for material science applications, an advantage above other techniques for the determination of the halogens and volatile elements such as As, Se, Sb and $\mathrm{Hg}$.

Participation in material science research provide considerable more analytical challenges than can be found in analysis of the traditional range of materials of siliceous origin such as soil, rocks and air particulate matter. It may often occur that the main matrix element is not silicon, and the induced activity of the impurities may have to be determined in the presence of a high induced activity of the principal component(s) of the material. It will require further development of detection systems for the processing of very high count rates-possibly with high resolution, fast scintillators, and application of new deconvolution algorithms for detection and quantification of small peaks at a high background of scattered photons.

Large sample NAA is unique in its kind, and applications may range from studies in which sample size reduction towards smaller test portions is undesirable of not even permitted. Applied on test portions of e.g. 10-100 g, LSNAA can provide an experimental verification of theories and (empirical) formulas for the minimum sample mass, e.g. in the food industry, mineral mining industry or material recycling industry. Applied at small research reactors, LS-NAA provides an opportunity to compensate for the low neutron fluence rate, thus also allowing samples to be counted at larger distance from the detector with contributes to improvement of the degree of accuracy. Another opportunity of (large sample) NAA to be rediscovered is with the use of enriched stable activable tracers in biology and industrial systems.

The outlook for applications in the applied fields is enduser driven: the analytical problem defined by the end-user and NAA having the capabilities for providing a solution. Such a situation may occur in each country with an NAA facility.

\section{Threats}

Sustainability of NAA laboratories depend on the availability and optimal use of resources. Traditional threats are the permanent shut-down of the research reactor or the absence of return of revenues for providing services. Shutdowns of reactors are partly related to the degree of its utilization and NAA is often the most intense user of the neutrons, especially in small and medium sized reactors. The size of the NAA program may be seriously hampered by shortages in staffing, lack of creativity and lack in automation. The latter is a technical detail that can relatively easy be solved. The lack of continuity in staffing and a missing adequate overlap period for retiring staff and newcomers may be the most serious threat to sustainability. Eventually this may lead to inability of continuing the NAA facility at the desired level of analytical and operational quality. The lack of inspiration to young people, e.g. students and trainees, is equally risky. It may be partly caused by a lack of creativity with respect to its usage, and not anticipating on developments and needs for analytical support in different and sometimes rapidly expanding fields of science. There are many countries in which national science foundations prioritize their grants for research that support such new applied sciences, whereas also the associated industry may finance projects. It may be argued that there are a few sustainable NAA laboratories that operate for decades in a specific field of applied science and which have reached an internationally renowned position. Still, a laboratory may loose its attractiveness for career making to young scientists if a mismatch occurs between the laboratory's focus and the inspiring new disciplines such as bio-nano-technology, as well as its country's needs, e.g. homeland security.

In addition, it should also not be underestimated that the absence of recently published textbooks may already have caused a negative image on the position and potentials of NAA. The excellent book by De Soete et al. [2] was published in 1972 and no other books with such a detailed description of the metrology of NAA have appeared since then. It may have created an image of a 'stuffy' and notdynamic science, which is not much inviting for career making. Consequently, there is an urgent need for a new comprehensive NAA textbook, comprising the current metrological expertise and providing examples of the use of NAA for solving today's problems in various fields of science.

\section{Conclusions}

The question, raised in the Introduction, “...does NAA can still advertise itself being a science with challenging innovative research on the principles and applications of the method, in such a way that will invite new people to step into it?..." can now be answered with "Yes". Yes, because NAA has some unique analytical features, such as the ability of bulk analysis of much larger portions than any other technique can handle. An outlook exists for even 3D mapping of the element amounts in such large samples.

And "Yes", since there are various developments in associated fields of science that, once tuned for and implemented in an NAA laboratory can be beneficial for further expansion of the strengths of the technique, and for overcoming its weaknesses. The sensitivity of NAA may be increased significantly by using scintillation detectors in 
combination with innovative spectrum deconvolution techniques. And "Yes", because new applications emerge in e.g. material science, nanotechnology and related to nuclear forensics, as well as for supporting many other sciences by studying the sampling error. Each new step requires, except for creative minds also concerted international action by collaboration between NAA laboratories to maintain momentum in the realization of such an innovation. If this will lead to sustainability depends on how the current expertise will be fostered for continuing the education of new generation of NAA practitioners.

Acknowledgment The author wants to thank Prof. Dr. Ir. Marcel de Bruin for the inspiring discussions on the subjects, outlined in this manuscript.

Open Access This article is distributed under the terms of the Creative Commons Attribution Noncommercial License which permits any noncommercial use, distribution, and reproduction in any medium, provided the original author(s) and source are credited.

\section{References}

1. Lyon WS, Ricci E, Ross HH (1970) Anal Chem 42:123R-129R

2. De Soete D, Gijbels R, Hoste J (1972) Neutron activation analysis. Wiley, New York

3. Girardi F, Guzzi G, Pauly J (1965) Anal Chem 37:1085-1092

4. Routti JT, Prussin SG (1969) Nucl Instrum Methods 72:125-142

5. Albert RD (1953) Rev Sci Instrum 24:1096-1101

6. Borg DC, Segel RE, Kienle JP, Campbell L (1961) Int J Appl Radiat Isot 11:10-29
7. Molnar GL (ed) (2004) Handbook of prompt gamma activation analysis: with neutron beams. Springer Verlag, Weinheim. ISBN 9781402013041

8. Westphal GP (1976) Nucl Instrum Methods 134:387-390

9. Downing RG, Maki JT, Fleming RF (1987) J Radioanal Nucl Chem 112:33-46

10. Bode P, Overwater RMW (1993) J Radioanal Nucl Chem 167: 169-176

11. Greenberg RR, Bode P, De Nadai Fernandes EA (2011) Spectrochim Acta B 66:193-241

12. De Bruin M, Korthoven PJM (1975) Radiochem Radioanal Lett 21:287-292

13. Sueki K, Kobayashi K, Sato W, Nakahara H, Tomizawa T (1996) Anal Chem 68:2203-2209

14. Nair AGC, Acharya R, Sudarshan K (2003) Anal Chem 75:4868-4874

15. Bedregal P, Mendoza P, Cohen IM, Montoya E (2011) J Radioanal Nucl Chem (accepted)

16. Belgya T, Kis A, Szentmiklósi L, Kasztovszky ZS, Kudejova P, Schulze R, Materna T, Festa G, Caroppi PA (2008) J Radioanal Nucl Chem 278:751-754

17. Peters NJ, Brockman JD, Robertson JD (2009) J Radioanal Nucl Chem 282:255-259

18. Koster-Ammerlaan MJJ, Bode P, Winkelman AJM (2011) J Radioanal Nucl Chem (accepted)

19. Meng LJ, Ramsden D (2000) IEEE Trans Nucl Sci 47:1329-1336

20. Sjoden GE, Detwiler D, LaVigne E, Baciak JE Jr (2009) IEEE Trans Nucl Sci 56:1329-1339

21. Pommé S (2011) Private communication

22. Bode P, Greenberg RR, De Nadai Fernandes EA (2009) Chimia 63:1-3

23. Calibration and Measurement Capabilities-CMCs. http://kcdb.bipm. org/appendixC/. Accessed 15 May 2011

24. Zeisler R, Tomlin BE, Murphy KE, Kučera J (2009) J Radioanal Nucl Chem 282:69-74

25. Verheijke ML, Jaspers HJJ, Hanssen JMG (1989) J Radioanal Nucl Chem 131:192-214 